\title{
Endoplasmic reticulum stress-induced apoptosis requires bax for commitment and Apaf-1 for execution in primary neurons
}

\author{
MI Smith ${ }^{1}$ and M Deshmukh ${ }^{\star, 1}$
}

\begin{abstract}
Apoptosis triggered by endoplasmic reticulum (ER) stress is associated with various pathophysiological conditions including neurodegenerative diseases and ischemia. However, the mechanism by which ER stress induces neuronal apoptosis remains controversial. Here we identify the pathway of apoptosis carried out in sympathetic neurons triggered to die by ER stressinducing agent tunicamycin. We find that ER stress induces a neuronal apoptotic pathway which upregulates BH3-only genes DP5 and Puma. Importantly, we show that ER stress commits neurons to die before cytochrome $c$ release and this commitment requires Bax activation and $\mathrm{c}$-jun $\mathrm{N}$-terminal kinase signaling. Furthermore, ER stress engages the mitochondrial pathway of death as neurons release cytochrome $c$ and Apaf-1 deficiency is sufficient to block apoptosis. Our findings identify a critical function of Bax in committing neurons to ER stress-induced apoptosis and clarify the importance of the apoptosome as the non-redundant caspase activation pathway to execute neuronal apoptosis in response to ER stress.
\end{abstract}

Cell Death and Differentiation (2007) 14, 1011-1019. doi:10.1038/sj.cdd.4402089; published online 12 January 2007

Endoplasmic reticulum (ER) stress occurs when the ability of the ER to properly fold proteins is overwhelmed or compromised, resulting in the activation of the unfolded protein response (UPR). ${ }^{1}$ Activation of UPR results in a global decrease in protein synthesis while increasing the production of ER chaperone proteins. However, under conditions of severe and prolonged ER stress, the UPR is unable to restore normal cellular function and apoptosis is triggered. Apoptosis induced by ER stress contributes to neuronal death seen in many neurodegenerative disorders as well as neuronal death following ischemia. ${ }^{2}$ Therefore, an understanding of the mechanism by which neurons undergo ER stress-induced apoptosis has important clinical significance.

Caspase proteases are the critical executioners of apoptosis in mammalian cells. ${ }^{3}$ Many death stimuli converge on the mitochondria to activate caspases by the intrinsic pathway. In this pathway, activation of $\mathrm{BH} 3-o n l y$ proteins serves to activate proapoptotic Bcl-2 family members Bax and Bak, ${ }^{4}$ which oligomerize in the mitochondrial membrane to induce the release of cytochrome $c .{ }^{5}$ Cytosolic cytochrome $c$ binds to Apaf-1 to form the apoptosome complex where caspase 9 is activated. Active caspase 9 cleaves and actives executioner caspases, such as caspase 3 , which then go on to cleave various target proteins resulting in the demise of the cell. ${ }^{5}$

The mechanism by which apoptosis is induced in response to ER stress has been examined in multiple cell types. Bax and Bak are important in this pathway, as mouse embryonic fibroblasts (MEFs) doubly deficient in Bax and Bak are resistant to ER stress-induced apoptosis. ${ }^{6,7}$ In addition to the established roles of Bax and Bak at the mitochondria, both are also found localized to the ER membrane and play an important role in $\mathrm{Ca}^{2+}$ regulation and release in response ER stress. $^{6-8}$

The discovery of an ER-localized caspase, caspase 12, has brought into question the mechanism by which ER stress activates caspases to carry out apoptosis. ${ }^{9}$ In vitro, active caspase 12 is able to cleave caspase 9 directly, which subsequently activates caspase 3 , potentially eliminating the requirement of the mitochondria and apoptosome to carry out ER stress-induced apoptosis. ${ }^{10,11}$ The lack of apoptosome involvement in ER stress-induced apoptosis is consistent with the observation that Sak2 cells, which are deficient in Apaf-1, are capable of activating caspases when treated with ER stress-inducing agents thapsigargin (TG) and brefeldin A. ${ }^{10}$ Likewise, C2C12 cells treated with tunicamycin (TU) or TG show caspase activation without any detectable release of cytochrome $c .^{11}$

Although caspase 12-deficient cells show resistance to ER stress stimuli, this resistance is mild. ${ }^{9}$ Additionally, human caspase 12 lacks protease activity, bringing into question the relative importance of the caspase 12 pathway in ER stressinduced apoptosis. ${ }^{12,13}$ Recent studies suggest that the mitochondrial pathway may be significant in either inducing or amplifying ER stress-induced apoptosis. First, in contrast to the data in $\mathrm{C} 2 \mathrm{C} 12$ cells, cytochrome $c$ is released in response to ER stress in a variety of other cell types. ${ }^{14,15}$ Second, a recent study found that the Apaf-1-deficient ETNA (murine embryonic telencephalic naïve) cell line and Apaf-1-deficient MEFs and embryonic cortical cells are resistant to ER stressinduced apoptosis. ${ }^{14}$ The disparities between these two sets

\footnotetext{
${ }^{1}$ Department of Cell and Developmental Biology and Neuroscience Center, University of North Carolina, Chapel Hill, NC, USA

*Corresponding author: M Deshmukh, 7109E Neuroscience Research Building, Box 7250, 105 Mason Farm Road, University of North Carolina, Chapel Hill, NC 27599, USA. Tel: + 919843 6004; Fax: + 919966 1050; E-mail: mohanish@med.unc.edu

Keywords: apoptosis; apaf-1; apoptosome; caspase; ER stress; Neurons; caspase

Abbreviations: CHOP, C/EBP homologous protein; ER, endoplasmic reticulum; MLK, mixed lineage kinase; NGF, nerve growth factor; TG, thapsigargin; TU, tunicamycin; UPR, unfolded protein response; MEFs, mouse embryonic fibroblasts; JNK, c-jun N-terminal kinase; ETNA, murine embryonic telencephalic naïve Received 26.6.06; revised 13.11.06; accepted 23.11.06; Edited by H Ichijo; published online 12.1.07
} 
of findings leave open the question as to whether mammalian cells, in particular primary post-mitotic neurons, undergo apoptosis in a mitochondria and apoptosome-dependent or -independent manner in response to ER stress.

In this study, we have examined the molecular pathway of ER stress-induced apoptosis in primary sympathetic neurons. Our data show that neuronal commitment to death in response to ER stress occurs before cytochrome $c$ release and is dependent on Bax activation and c-jun $\mathrm{N}$-terminal kinase (JNK) signaling. Importantly, we show clear evidence that upon commitment, neurons engage the mitochondrial pathway and utilize the apoptosome as a non-redundant mechanism to induce apoptosis.

\section{Results}

TU-induced ER stress results in caspase-dependent apoptosis in neurons. TU, a $\mathrm{N}$-glycosylation inhibitor, induces death in many cell types including sympathetic and hippocampal neurons. ${ }^{15,16}$ Sympathetic neurons treated with $2.5 \mu \mathrm{M}$ TU exhibited a robust increase in the levels of ER stress-induced transcription factor C/EBP homologous protein (CHOP) and the ER resident chaperone BiP (Figure $1 \mathrm{a}$ and $\mathrm{b}$ ). Additionally XBP-1 became spliced, indicating that the ER stress sensor IRE1 was activated (Figure 1b). Together, these results show that a vigorous ER stress response was being induced.

To determine the time course with which TU kills sympathetic neurons, neuronal viability was assessed, by cell morphology, at various time points following treatment. Following $36 \mathrm{~h}$ of TU treatment, approximately $70 \%$ of neurons were still viable. However, by $60 \mathrm{~h}$ virtually all neurons in the culture were dead (Figure 1c and $d$ ). This method of measuring viability was confirmed by use of the 1-4,5-dimethylthiazol-2-yl)-2,5-diphenyltetrazolium bromide (MTT) assay and correlates well with other cell survival assays such as trypan blue exclusion and staining with calcein AM (Supplemental Figure 1A). ${ }^{17}$ Importantly, TU-induced neuronal death is caspase dependent as it was completely inhibited by the pan caspase inhibitor zVAD-fmk $(50 \mu \mathrm{M})$ (Figure 1c and d). zVAD-fmk appeared to have no affect on the ER stress response itself as levels of BiP and XBP-1 splicing were unaffected by the addition of zVAD-fmk to TU-treated cultures (Figure 1b). These results show that TU induces ER stress and caspase-dependent death in neurons.

Neurons activate JNK signaling which is required for apoptosis in response to ER stress. JNK is known to be activated following ER stress by the IRE1-TRAF2-ASK1 pathway. ${ }^{18,19}$ However, in sympathetic neurons, proapoptotic stimuli have been shown to activate JNK via mixed lineage kinases (MLK). ${ }^{20,21}$ In these neurons, MLK activation by Cdc42/Rac is thought to phosphorylate MEKK4/7 which subsequently phosphorylates and activates JNK signaling. ${ }^{22,23}$ JNK activation leads to the upregulation, phosphorylation and activation of the transcription factor c-Jun which is essential for sympathetic neuronal apoptosis in response to nerve growth factor (NGF) withdrawal. ${ }^{24,25}$ We examined whether the JNK pathway was activated in sympathetic neurons in response to ER stress by using immunohistochemistry to assess the phosphorylation state of c-Jun. Although untreated neurons showed virtually no phospho-c-Jun staining, by $18 \mathrm{~h}$ neurons treated with TU showed high levels of nuclear localized phosphorylated a
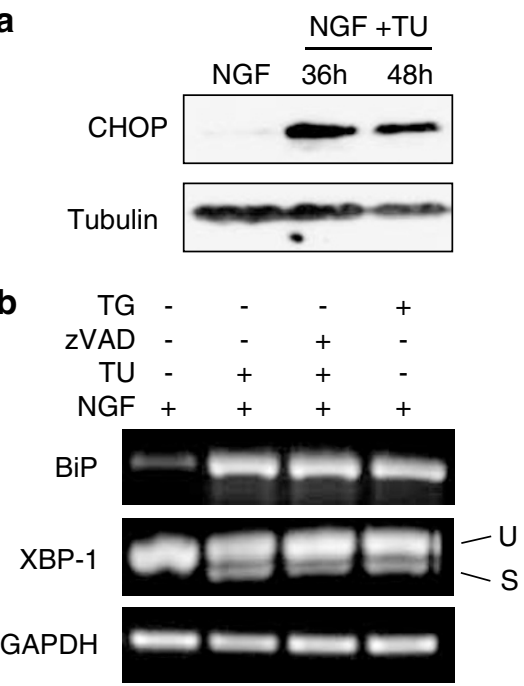

C

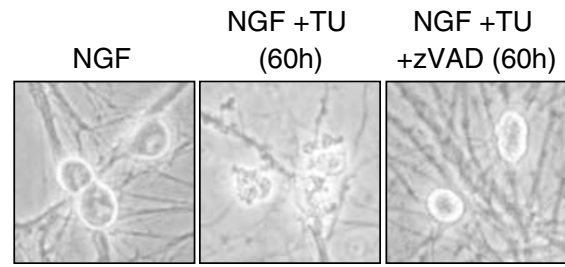

d

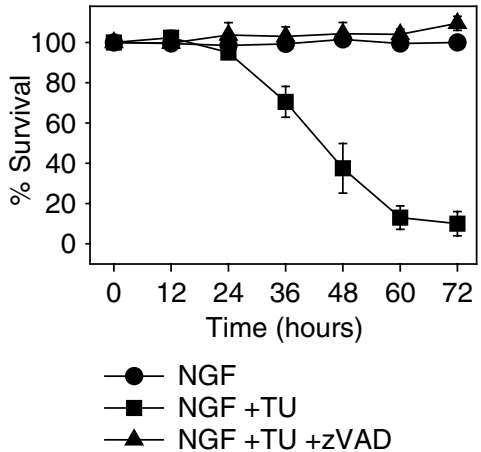

Figure 1 TU causes ER stress-induced apoptosis in sympathetic neurons. Cultures of sympathetic neurons were untreated (NGF), treated with $2.5 \mu \mathrm{M}$ TU (NGF + TU) or treated with $2.5 \mu \mathrm{M} \mathrm{TU}$ and $50 \mu \mathrm{M} \mathrm{zVAD}$-fmk (NGF + TU + zVAD). (a) Levels of CHOP, a marker of ER stress, were examined by Western blot of whole-cell lysate from sympathetic neurons collected 36 or $48 \mathrm{~h}$ after $2.5 \mu \mathrm{M}$ TU treatment or untreated neurons. Tubulin serves as a loading control. (b) mRNA levels of BiP and the spliced form of XBP-1 ( $\mathrm{U}$ - unspliced, $\mathrm{S}$ - spliced), both indicators of ER stress, were examined by RT-PCR after $24 \mathrm{~h}$ of treatment. Cultures were also treated with an alternate ER stress inducer $10 \mu \mathrm{M} \mathrm{TG}$ (NGF + TG). GAPDH serves as a control. Data are representative of $n \geqslant 2$ separate experiments per time point. (c) Photographs were taken $60 \mathrm{~h}$ following treatment. (d) Survival of sympathetic neurons was accessed by cell morphology at the indicated time points. Data are means \pm S.E.M. from $n \geqslant 3$ separate experiments per time point 
c-Jun, which peaked by $24 \mathrm{~h}(76 \%)$ and were still elevated at $36 \mathrm{~h}$ (Figure 2a).

To determine if the MLK-mediated JNK pathway was required for ER stress-induced apoptosis, we treated sympathetic neurons with TU in the presence of a MLK inhibitor, CEP-11004. ${ }^{21,26}$ Following $48 \mathrm{~h}$ of treatment, at a time when only about $10 \%$ of the TU-treated neurons were alive, greater than $85 \%$ of the TU and CEP-11004-treated neurons remained viable (Figure 2b). CEP-11004 was also protective against TG-induced neuronal death (Supplemental Figure 1B). CEP-11004 did not affect the ER stress response, as mRNA levels of BiP and XBP-1 splicing were unaffected by the addition of CEP-11004 to TU-treated cultures (Figure 2c). However, CEP-11004 was fully capable of blocking the phosphorylation of c-jun in TU-treated neurons (Figure 2d). These results indicate that the MLK-mediated JNK pathway is activated during ER stress-induced neuronal apoptosis and its inhibition is sufficient to block or prolong death.

BH3-only genes DP5 and Puma are upregulated independently of MLK signaling during ER stressinduced neuronal apoptosis. BH3-only proteins are responsible for binding to and activating proapoptotic Bax and Bak in response to diverse apoptotic stimuli. ${ }^{4} \mathrm{BH} 3-$ only proteins themselves can be activated by transcriptional or post-translational mechanisms. For example, following NGF withdrawal in sympathetic neurons, BH3-only proteins Bim, DP5 and Puma become transcriptionally upregulated in part via the JNK pathway (Figure $3 \mathrm{c}$ and d) ${ }^{4}$

We examined whether BH3-only proteins were transcriptionally upregulated in primary sympathetic neurons treated with TU. Our results show that both DP5 and Puma are transcriptionally induced as early as $12 \mathrm{~h}$ by ER stress whereas levels of Noxa and Bim were not significantly altered (Figure 3a and b).

We next examined whether MLK signaling was important for the transcriptional upregulation of these $\mathrm{BH}$-only genes in ER stress-induced neuronal apoptosis. Surprisingly, CEP11004 addition did not block the transcriptional increase in DP5 and Puma seen after TU treatment in these neurons (Figure $3 \mathrm{c}$ and d). These results indicate that the mechanism by which $\mathrm{BH} 3$-only genes are transcriptionally upregulated during ER stress-induced apoptosis is MLK independent.

Bax deficiency inhibits ER stress-induced apoptosis in neurons, allowing for long-term survival. Bax/Bak double-knockout MEFs have been shown to be highly resistant to many apoptotic insults including ER stress. ${ }^{6,7}$ Even though Bax and Bak act redundantly to induce apoptosis in fibroblasts, sympathetic neurons do not a

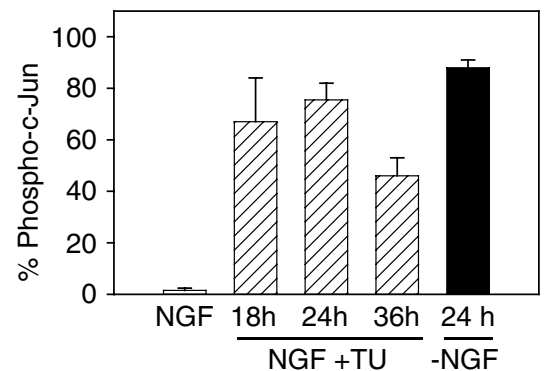

b

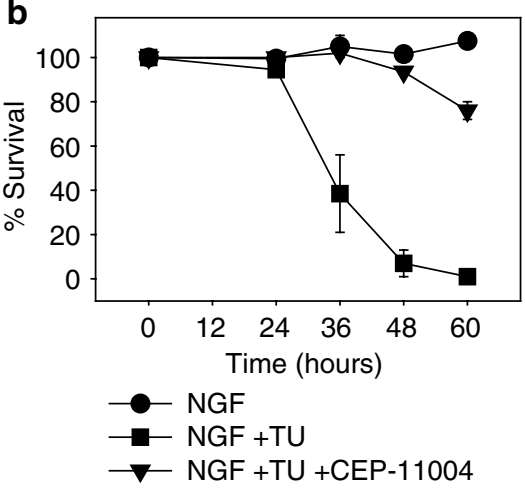

C

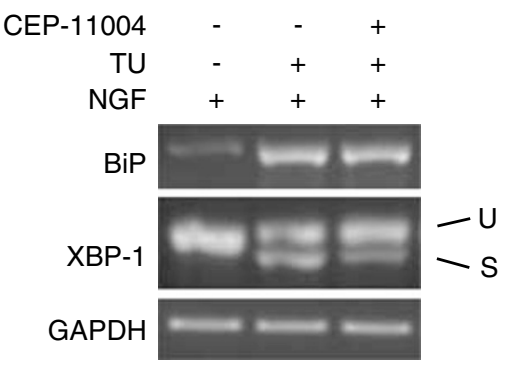

d

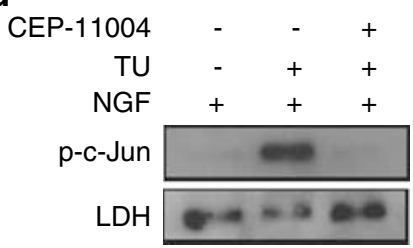

Figure 2 JNK signaling is required for ER stress-induced neuronal apoptosis. (a) Presence of active, phosphorylated c-Jun was examined by immunofluorescence in sympathetic neurons that were untreated (NGF) or treated with $2.5 \mu \mathrm{M}$ TU for 18, 24 or $36 \mathrm{~h}$ (NGF + TU). Cultures deprived of NGF for $24 \mathrm{~h}$ ( $-\mathrm{NGF}$ ) served as a positive control for c-Jun phosphorylation. Neurons were also stained with Hoechst to detect nuclei and the percentage of neurons with Phospho-c-Jun was quantified as the percentage of nuclei, which also had Phospho-c-Jun-positive staining. Data are means \pm S.E.M. from $n \geqslant 3$ separate experiments per treatment. (b) Sympathetic neurons were either untreated (NGF), treated with $2.5 \mu \mathrm{M}$ TU (NGF + TU) or treated with $2.5 \mu \mathrm{M}$ TU and $3 \mu \mathrm{M}$ CEP-11004 (NGF + TU + CEP-11004), a MLK inhibitor. Survival was accessed by cell morphology at the indicated times. Data are means \pm S.E.M. from $n \geqslant 3$ separate experiments per time point. (c) mRNA levels of BiP and the spliced form of XBP-1 ( $\mathrm{U}$ - unspliced, $\mathrm{S}$ - spliced), both indicators of ER stress, were examined by RT-PCR after $24 \mathrm{~h}$ of treatment. GAPDH serves as a control. Data are representative of $n \geqslant 2$ separate experiments per time point 
a

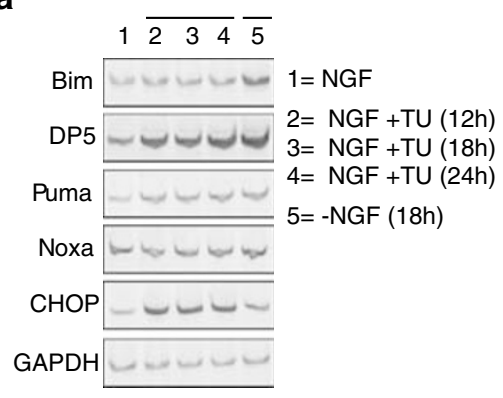

c

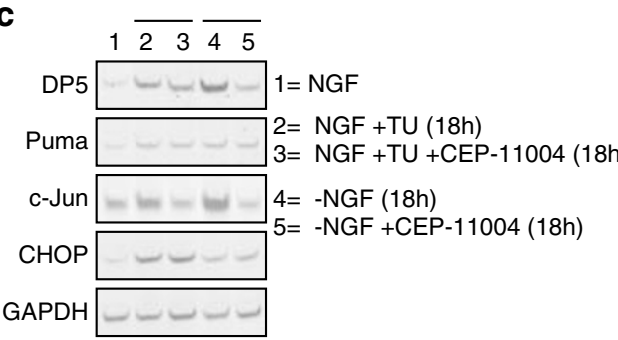

b

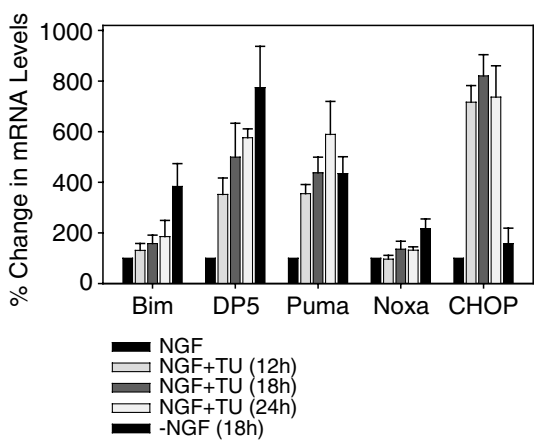

d

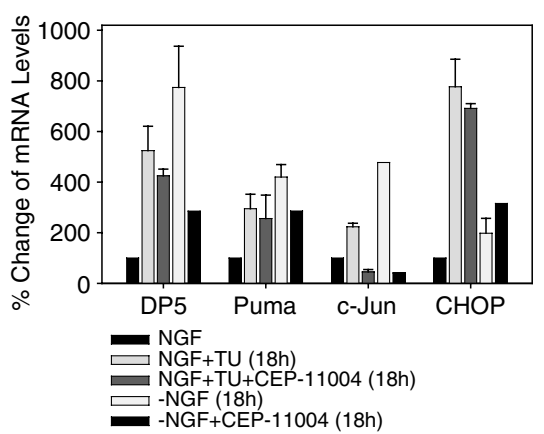

Figure 3 BH3-only proteins Puma and DP5 are transcriptionally upregulated independently of MLK signaling following ER stress in sympathetic neurons. (a) Cultured sympathetic neurons were untreated (NGF), treated with TU for 12, 18 or $24 \mathrm{~h}$ (NGF + TU) or deprived of NGF for $18 \mathrm{~h}$ (-NGF) before collection of total RNA. All cultures were treated with $50 \mu \mathrm{M}$ zVAD-fmk to prevent cell loss. RT-PCR was carried out with primers for the indicated proteins. CHOP levels were examined to ensure ER stress was being induced. GAPDH serves as a loading control. (b) Quantification of (a) normalized to GAPDH levels. Data are means \pm S.E.M. from $n \geqslant 3$ separate experiments per treatment. (c) Cultured sympathetic neurons were untreated (NGF), treated with $2.5 \mu \mathrm{M}$ TU for $18 \mathrm{~h}$ in the absence (NGF + TU) or presence of $3 \mu \mathrm{M}$ CEP-11004 (NGF + TU + CEP11004) or deprived of NGF for $18 \mathrm{~h}$ in the absence (-NGF) or presence of $3 \mu \mathrm{M}$ CEP-11004 (-NGF + CEP11004) before collection of total RNA. All cultures were treated with $50 \mu \mathrm{M}$ zVAD-fmk to prevent cell loss. RT-PCR was carried out with primers for the indicated proteins. CHOP levels were examined to ensure ER stress was being induced. GAPDH serves as a loading control. (d) Quantification of (c) normalized to GAPDH levels. Data are means \pm S.E.M. from $n \geqslant 3$ separate experiments per treatment

express full-length Bak. $^{27}$ As a result, Bax-deficient sympathetic neurons are resistant to death following NGF deprivation and DNA damage. ${ }^{28,29}$ Therefore, we examined whether Bax deficiency alone was capable of preventing ER stress-induced apoptosis in sympathetic neurons. Our results show that Bax-deficient neurons remained viable long after the wild-type neurons had undergone apoptosis in response to both TU and TG (Figure 4a and b, Supplementary Figure 4).

To determine whether Bax deficiency was simply delaying neuronal death, we maintained the Bax-deficient neurons with or without TU for 11 days. Remarkably, Bax-deficient neurons exhibited decreased cell volume but no morphological signs of apoptosis even with prolonged exposure to TU (Figure 4c). This resistance to ER stress did not appear to be owing to decreased activation of IRE1, as reported for Bax/Bakdeficient MEFs, ${ }^{30}$ as Bax-deficient neurons were still able to splice the IRE1 target, XBP-1 (Figure 4d). Together, these results show that $B a x$ is essential for $E R$ stress-induced apoptosis in neurons and that in the absence of Bax, TUtreated neurons can survive long term.

ER stress commits neurons to die early before cytochrome $c$ release, and requires $B a x$ and JNK activation. Commitment to death is the point in the apoptotic pathway at which neurons can no longer recover and eventually die, even with the removal of the apoptotic stimuli. Defining the point of commitment is important from a therapeutic perspective as long-term neuronal survival is expected if death is blocked upstream of commitment, whereas blocking downstream of commitment will only serve to delay death. In NGF deprived neurons, commitment to death occurs coincident with the time of cytochrome $c$ release. ${ }^{31}$ Our data show that the ER stress-induced time course of cytochrome $c$ release corresponded very well to the time of death (Figure 5a). ${ }^{31}$ However, unlike NGF deprivation, TU-treated neurons were committed to die significantly before cytochrome $c$ release. For example, following $24 \mathrm{~h}$ of TU treatment whereas only $20 \%$ of neurons had released cytochrome $c$, greater than $80 \%$ of neurons had already become committed to die (Figure 5a).

To narrow down the point of commitment in ER stressinduced apoptosis, we examined commitment in Bax-deficient neurons. If commitment occurs upstream of Bax activation, then the Bax-deficient neurons are expected to eventually undergo cell death in this assay. Our results show that in the absence of Bax, sympathetic neurons do not become committed to die in response to ER stress. Even after $120 \mathrm{~h}$ of TU treatment, the Bax-deficient neurons failed to be committed to death (Figure $5 \mathrm{~b}$ ). These results identify a critical role of Bax for commitment to death in neurons undergoing ER stress-induced apoptosis. 
a

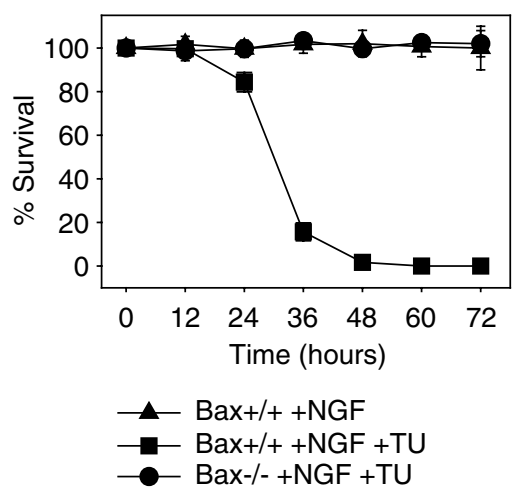

b

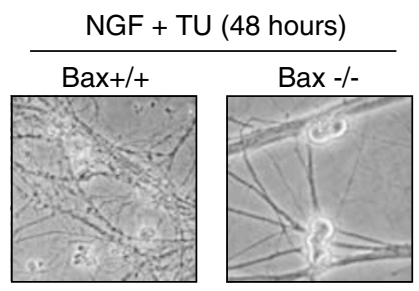

c

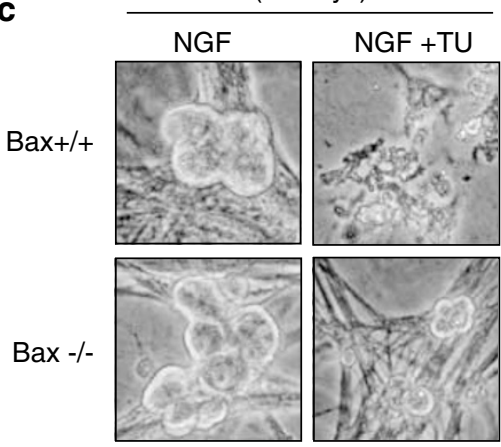

d

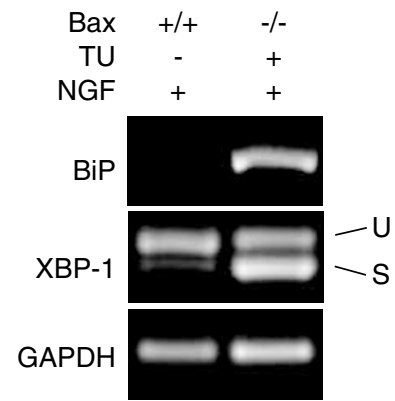

Figure 4 Bax deficiency blocks ER stress-induced apoptosis allowing for long-term survival of sympathetic neurons. (a) Sympathetic neurons from Bax-deficient mice (Bax-/-) and their wild-type littermates (Bax $+/+$ ) were untreated (NGF) or treated with $2.5 \mu \mathrm{M} \mathrm{TU}$ (NGF+TU). Neuronal survival was determined by cell morphology at each of the indicated time points. Data are means \pm S.E.M. from $n \geqslant 3$ separate experiments per time point. (b) Photographs of wild-type and Bax-deficient sympathetic neurons treated with $2.5 \mu \mathrm{M}$ TU for $48 \mathrm{~h}$. (c) Photographs of wild-type (Bax $+/+)$ and Bax-deficient (Bax-/-) sympathetic neurons untreated or treated with $2.5 \mu \mathrm{M}$ TU for 11 days. Fresh media containing TU was replenished every 3 days to ensure a sustained ER stress response. Photographs were taken at the same magnification. (d) Sympathetic neurons from Bax-deficient (Bax-/-) and wild-type (Bax $+/+$ ) mice were untreated (NGF) or treated with $2.5 \mu \mathrm{M}$ TU (NGF + TU) for $24 \mathrm{~h}$. Using RT-PCR, the spliced form of XBP-1 (U - unspliced, S - spliced) was examined as an indicator of IRE1 activity, and level of BiP was examined as a marker of ER stress. GAPDH serves as a control. Data are representative of $n \geqslant 2$ separate experiments per time point

A recent report determined that Bax and Bak at the ER are critical for JNK activation following ER stress in fibroblasts. ${ }^{30}$ Therefore, we examined the importance of JNK signaling in neuronal commitment to death in ER stress. Sympathetic neurons were treated with TU for 12, 24 and $36 \mathrm{~h}$ following which the drug was washed out and either fresh media alone or fresh media containing the MLK inhibitor CEP-11004 was added during the recovery period. Although CEP-11004 addition $12 \mathrm{~h}$ after TU treatment was still capable of inhibiting the commitment to death, by $24 \mathrm{~h}$ after TU treatment CEP11004 could no longer inhibit commitment in the majority of neurons (Figure 5c). Similar results were also obtained with the JNK inhibitor SP600125 (Figure 5d). These results point to the importance of JNK signaling-dependent events, which occur between 12 and $24 \mathrm{~h}$ following TU treatment, in committing neurons to ER stress-induced apoptosis.

Apaf-1 is required for neurons to undergo ER stressinduced apoptosis. Although the role of the Apaf-1dependent apoptosome pathway is well established in many models of apoptosis, its specific importance in the ER stress-induced apoptotic pathway is controversial. ${ }^{32}$ To determine if the cytochrome $c$-mediated apoptosome pathway is required to carry out ER stress-induced neuronal apoptosis, we examined the effects of ER stress on sympathetic neurons isolated from Apaf-1-deficient mice. First, we ensured that Apaf-1-deficient neurons were capable of releasing cytochrome $c$ in response to TU. Our results show that by $48 \mathrm{~h}$ cytochrome $c$ was released from mitochondria in both wild-type and Apaf-1-deficient neurons in response to TU treatment (Figure 6a and b).

Next we examined whether Apaf-1 was required for ER stress-induced apoptosis in sympathetic neurons. In contrast to wild-type neurons, which were dead by $48 \mathrm{~h}$, Apaf-1deficient neurons remained alive even at $96 \mathrm{~h}$ of TU treatment (Figure 6c). These results identify an essential requirement of Apaf-1 in neurons undergoing apoptosis in response to TU.

Together, these results demonstrate that ER stress engages Bax-dependent-cytochrome $c$ release and requires the apoptosome pathway of caspase activation to carry out neuronal apoptosis.

\section{Discussion}

In this study, we utilized the model of primary sympathetic neurons to examine the molecular pathway engaged by ER stress-induced apoptosis in post-mitotic neurons. First, we report that ER stress-induced neuronal apoptosis requires JNK signaling. TU induced the phosphorylation and upregulation of c-Jun in neurons and blocking this pathway with the MLK inhibitor CEP-11004 or the JNK inhibitor SP600125 
a

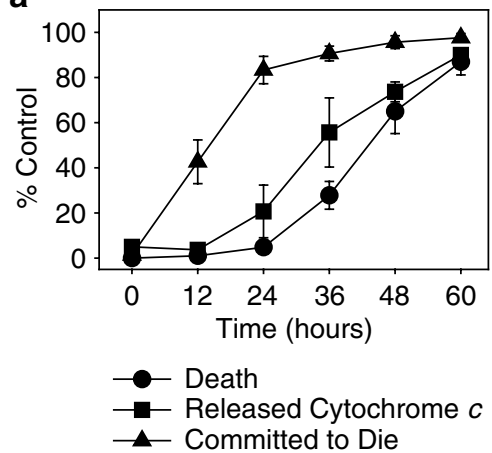

C

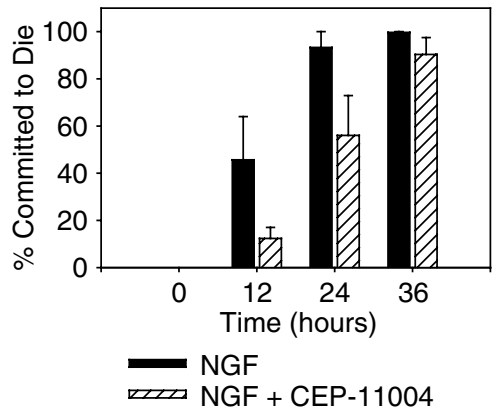

b

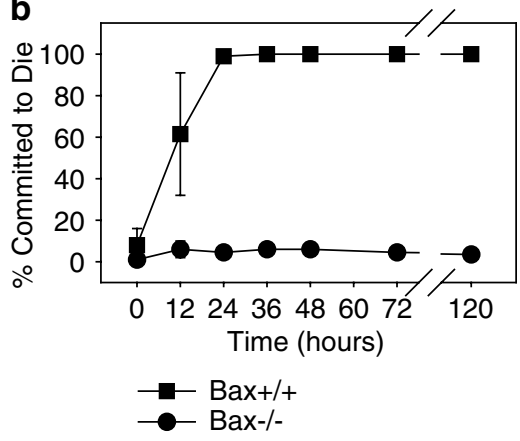

d

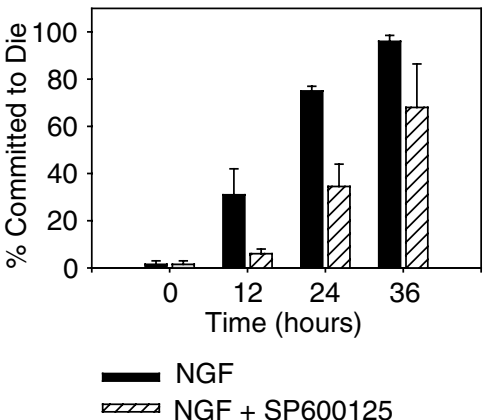

Figure 5 ER stress-induced commitment to death occurs well before cytochrome $c$ release and is blocked by Bax deficiency and by inhibiting JNK signaling. (a) Sympathetic neurons were treated with $2.5 \mu \mathrm{M}$ TU and the death, release of cytochrome $c$ and commitment to die were quantified. Cell morphology was assessed to determine the percent of death at the indicated time points. Cytochrome $c$ release was determined by immunofluorescence. These immunofluorescence studies were carried out on cultures treated with $50 \mu \mathrm{M}$ zVAD-fmk to prevent cell loss. Neurons were also stained with Hoechst to detect nuclei and the percentage of neurons, which had released cytochrome $c$ was measured as a percentage of cells which had nuclei but lacked intact punctate cytoplasmic cytochrome $c$ staining. Commitment to die studies were carried out as indicated and explained in the results and Materials and Methods sections. The percent of neurons committed to die was determined as the percent of the cells lost after the recovery period (5-7 days) relative to those present before the treatment period (length of the treatment period is as indicated on the graph). Data are means \pm S.E.M. from $n \geqslant 3$ separate experiments per time point. (b) Sympathetic neurons isolated from Bax-deficient mice (Bax $-I-$ ) or their wild-type littermates (Bax $+I+$ ) were exposed to $2.5 \mu \mathrm{M} \mathrm{TU}$ and commitment was determined as described in (a). Data are means \pm S.E.M. from $n \geqslant 3$ separate experiments per time point. (c) Sympathetic neuronal cultures were exposed to $2.5 \mu \mathrm{M} \mathrm{TU}$ and commitment studies were carried out as described in (a) with the exception that neurons were either saved with NGF containing media or NGF containing media with $3 \mu \mathrm{M}$ CEP-11004. Data are means \pm S.E.M. from $n \geqslant 3$ separate experiments per time point. (d) Sympathetic neuronal cultures were exposed to $2.5 \mu \mathrm{M}$ TU and commitment studies were carried out as described in (a) with the exception that neurons were either saved with NGF containing media or NGF containing media with $10 \mu \mathrm{M}$ SP600125. Data are means \pm S.E.M. from $n \geqslant 2$ separate experiments per time point
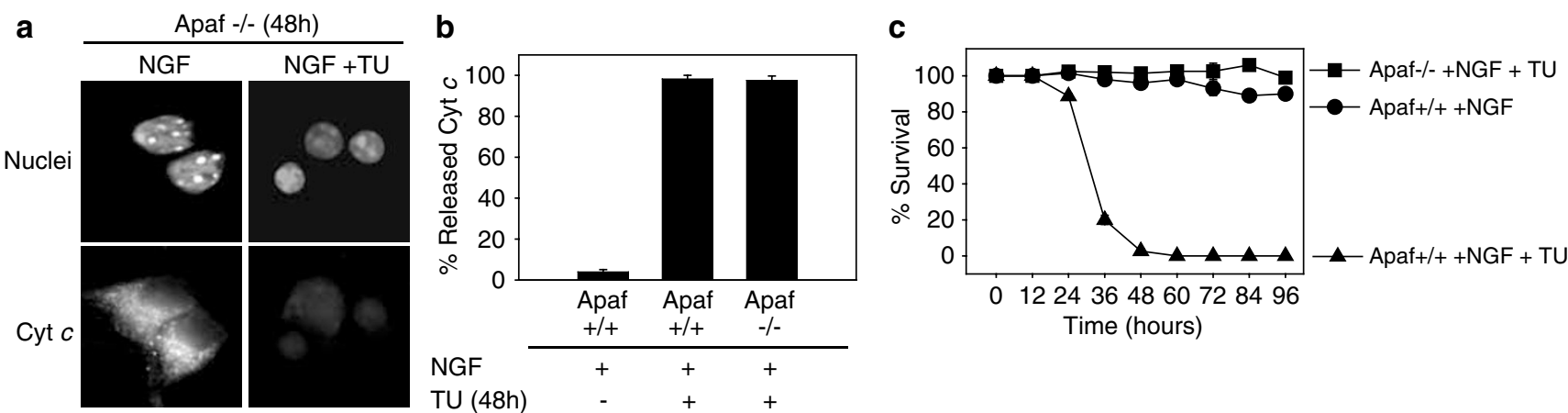

Figure 6 Apaf-1-deficient sympathetic neurons are able to release cytochrome $c$ but do not undergo ER stress-induced apoptosis. (a) Apaf-1-deficient sympathetic neurons (Apaf-/-) were untreated (NGF) or treated with $2.5 \mu \mathrm{M}$ TU (NGF + TU) for $48 \mathrm{~h}$. Neurons were also stained with Hoechst to detect nuclei. (b) Apaf-1-deficient sympathetic neurons (Apaf-I-) and their wild-type littermates (Apaf $+/+$ ) were untreated (NGF) or treated with $2.5 \mu \mathrm{M} \mathrm{TU}$ (NGF + TU) for $48 \mathrm{~h}$ in the presence of $50 \mu \mathrm{M}$ zVAD-fmk. Neurons were also stained with Hoechst to detect nuclei and the percentage of neurons, which had released cytochrome $c$ was measured as those which had nuclei but lacked intact punctuate cytoplasmic cytochrome $c$ staining. Data are means \pm S.E.M. from $n \geqslant 3$ separate experiments per treatment. (c) Apaf-1-deficient sympathetic neurons (Apaf-I-) and their wild-type littermates (Apaf $+/+$ ) were untreated (NGF) or treated with $2.5 \mu \mathrm{M}$ TU (NGF + TU). Cell survival was accessed by morphology at the indicated time points. Data are means \pm S.E.M. from $n \geqslant 3$ separate experiments per time point 
prevented apoptosis (Figures 2, 3, 5). Second, we identify Puma and DP5 as two BH3-only genes that are transcriptionally activated in response to ER stress (Figure 3 ). This increase in Puma and DP5 occurred even in the presence of CEP-11004 (Figure 3). Third, in response to ER stress, neurons release cytochrome $c$ (Figure 5a). However, neurons became committed to die long before the point of cytochrome $c$ release and this commitment requires both Bax activation and JNK signaling (Figure 5). Thus, Bax deletion alone is able to block ER stress-induced apoptosis long term in both cell survival and commitment studies (Figures 4 and 5b, Supplementary Figure 4). Finally, we show that Apaf-1-deficient neurons are remarkably resistant to ER stress-induced apoptosis (Figure 6c). These results identify an essential and non-redundant role of the Apaf-1-dependent apoptosome pathway in mediating ER stress-induced apoptosis in neurons.

ER stress induces the activation of JNK signaling and the transcriptional upregulation of $\mathrm{BH} 3-$ only proteins during neuronal apoptosis. Our results show that TU induced an early and sustained increase in JNK-dependent phosphorylation of c-Jun in neurons (Figure 2a and d). JNK signaling could be activated at the ER membrane though IRE1-TRAF2-ASK1 pathway or through the MLK pathway that is known to become activated in these sympathetic neurons after NGF deprivation. ${ }^{18-21}$ Studies utilizing ASK1deficient mice have also demonstrated the importance of ASK1 in ER stress-induced apoptosis in telencephalic neurons. ${ }^{19}$ We find that an increase in JNK signaling was required for ER stress-induced apoptosis in sympathetic neurons as the addition of SP600125 (JNK inhibitor) or CEP11004 (MLK inhibitor) blocked apoptosis (Figures 2b, 5c and d). Interestingly, CEP-11004 addition did not protect against TU-induced death in primary fibroblasts (Supplementary Figure 2). These results point to the importance of MLK signaling mediating JNK activation in sympathetic neurons undergoing apoptosis with ER stress, just as seen after NGF deprivation. Therefore, it is likely that both these pathways contribute to JNK activation during ER stress-induced apoptosis in neurons.

JNK signaling is important in other models of neuronal apoptosis as well. ${ }^{20,25,33}$ For example, during NGF deprivation-induced apoptosis, JNK signaling appears to be important for the transcriptional upregulation of $\mathrm{BH} 3-$ only proteins DP5/Harakiri and Bim. ${ }^{4}$ We found DP5 and Puma levels were induced, however, Bim and Noxa levels did not change significantly after TU treatment in neurons (Figure 3a and b). Recent studies with $\mathrm{DP} 5^{34}$ and $\mathrm{Puma}^{35}$ knockout mice have shown that these $\mathrm{BH}$-only proteins contribute to apoptosis in sympathetic neurons. Importantly, addition of the MLK inhibitor CEP-11004, which blocks the upregulation and phosphorylation of c-Jun, did not block the increase in DP5 and Puma after ER stress (Figure $3 c$ and $d$ ). These results are consistent with the observation that $\mathrm{BH} 3-$ only proteins can be activated via JNK-dependent and JNK-independent pathways during sympathetic neuronal apoptosis. ${ }^{4}$

Our results showing the transcriptional upregulation of Puma following ER stress are in agreement with other studies. ${ }^{15,36}$ Although Puma was initially identified as a p53-regulated $\mathrm{BH} 3$-only gene, p53-deficient neurons were still able to upregulate Puma and did not show any resistance to ER stress-induced apoptosis (Supplementary Figure 3). Thus, whereas transcription factors c-Jun and p53 do not appear to be important for ER stress-induced DP5 and Puma upregulation, this upregulation could be mediated by alternative transcription factors such as $\mathrm{FOXO3a}$, which is known to be activated in neurons in response to other apoptotic stimuli. $^{4}$

ER stress commits neurons to die before cytochrome $c$ release and requires Bax. Neurons exposed to ER stress become committed to die long before they release cytochrome $c$ (Figure 5a). These results are in contrast to NGF deprivation, in which commitment to death occurs coincident with the mitochondrial release of cytochrome $c .^{31}$ In this respect, ER stress appears similar to DNA damage which also commits neurons to die early, before cytochrome $c$ release. ${ }^{29}$ As DNA damage commits neurons to die before Bax function at the mitochondria, Bax-deficient neurons still become committed to die. ${ }^{29}$ In striking contrast, Bax-deficient neurons exposed to ER stress fail to become committed to die (Figure $5 b$ ). These results show that during ER stress, Bax activation is an absolute requirement for neuronal commitment to death. Although Bax function at the mitochondria is well characterized, Bax has also been localized to the ER in fibroblasts and cell lines. ${ }^{6-8}$ Therefore, this difference in the requirement of Bax for neuronal commitment to death in response to ER stress but not DNA damage could be indicative of a critical function of Bax at the ER in neurons.

The Bax-deficient neurons that are unable to be committed to die in the presence of TU exhibit no morphological signs of degeneration even after 11 days of sustained exposure to TU (Figure 4c). These neurons are smaller than untreated neurons presumably owing to the global inhibition of protein synthesis that is seen after ER stress in mammalian cells (Figure 4c). ${ }^{1}$ Although it is surprising that these neurons can survive long term under these conditions, it is possible that they do so by utilizing autophagy which is known to provide energy in conditions of nutrient deprivation. ${ }^{37}$

The Apaf-1-dependent apoptosome pathway is required for neurons to undergo ER stress-induced apoptosis. There is significant debate about the importance of caspase 12 and the role of an Apaf-1-independent pathway in ER stress-induced apoptosis. Others have proposed that caspase 12 , which is activated at the ER, directly cleaves and activates caspase 9 which is then free to go on to cleave and activate caspase $3 .^{10,11}$ This implies that the apoptosomal components and therefore the apoptosome itself is not required for ER stress-induced apoptosis.

Here we show that in response to ER stress, sympathetic neurons release cytochrome $c$ and that Apaf- 1 is required for apoptosis (Figure 6). Thus, ER stress-induced apoptosis is not able to bypass the apoptosome, nor does the apoptosome act simply as a feedback amplification loop following caspase activation by an apoptosome-independent pathway. These finding are consistent with a recent study focusing on ETNA cells (neuronal precursor cell line) which also found the 
apoptosome pathway to be key to ER stress-activated apoptosis. ${ }^{14}$ Additionally, we find that a zVAD-fmk inhibitable caspase, such as caspase $12,{ }^{9}$ does not act upstream of the mitochondrial release of cytochrome $c$ during ER stressinduced apoptosis, as addition of zVAD-fmk did not block cytochrome $c$ release in response to TU treatment (Figure 5a). In agreement with this finding, recent studies have indicated that caspase 12 is a downstream target of caspases $3^{38}$ and that caspase 12 and human caspase 4 are dispensable for ER stress-induced apoptosis. ${ }^{39}$ Therefore, caspase 12 is not likely to play an essential role as a link between the ER and mitochondrial to release cytochrome $c$ in sympathetic neurons.

Together, the data presented in this study identify key components of the ER stress-induced apoptotic pathway in neurons. In particular, these results demonstrate the essential and non-redundant function of the Apaf-1-dependent mitochondrial pathway in this model. Our results also point to Bax inhibition as a viable strategy for preventing neuronal death in pathological models of ER stress, as it would block the commitment to death point preventing not only apoptosis but other forms of death, allowing for long-term survival.

\section{Materials and Methods \\ Reagents. All reagents were purchased from Sigma-Aldrich or Fisher scientific, unless otherwise stated. Collagenase and trypsin were purchased from Worthington Biochemical Corporation. zVAD-fmk purchased from Enzyme Systems Products (MP Biomedicals). CEP-11004 was a kind gift from Cephalon Inc. (Frazer, PA). Aphidicolin was purchased from Sci-Quest-AG Scientific. Protease inhibitor cocktail was purchased from Roche.}

Sympathetic neuronal and fibroblast cultures. Primary sympathetic neurons were isolated from superior cervical ganglia of post-natal day zero to one (P0-P1) mice as previously described for rats. ${ }^{31}$ Briefly, dissected ganglia were treated with $1 \mathrm{mg} / \mathrm{ml}$ collagenase, followed by $2.5 \mathrm{mg} / \mathrm{ml}$ trypsin for $30 \mathrm{~min}$ each at $37^{\circ} \mathrm{C}$. Ganglia were triturated and dissociated and plated on collagen-coated dishes at a density of 60000 cells per well for protein lysates or RNA extraction and 10000 cells per well for survival counts and immunofluorescence experiments. Neurons were maintained in media containing MEM with Earle's salts supplemented with $50 \mathrm{ng} / \mathrm{ml} \mathrm{NGF}, 10 \%$ FBS, $2 \mathrm{mM}$ glutamine, $100 \mu \mathrm{g} / \mathrm{ml}$ penicillin, $100 \mu \mathrm{g} / \mathrm{ml}$ streptomycin, $20 \mu \mathrm{M}$ fluorodeoxyuridine, $20 \mu \mathrm{M}$ uridine and $3.3 \mu \mathrm{g} / \mathrm{ml}$ aphidicolin. Experiments were preformed on cells $4-5$ days after plating. For NGF deprivation, cultures were rinsed twice with medium lacking NGF followed by addition of goat anti-NGF-neutralizing antibody to this NGF-free media. Other conditions required the addition of $2.5 \mu \mathrm{M}$ TU, $50 \mu \mathrm{M}$ zVAD-fmk or $3 \mu \mathrm{M}$ CEP-11004. In all of our studies we used a dosage of $2.5 \mu \mathrm{M}$ TU. This dosage was based on a doseresponse we preformed (data not shown) and falls well within the range used in various studies of cultured cells including sympathetic neurons. ${ }^{15,16}$

Primary MEFs were isolated from PO mice. The dorsal skin was removed and treated with $1 \mathrm{mg} / \mathrm{ml}$ collagenase, and then with $2.5 \mathrm{mg} / \mathrm{ml}$ trypsin for $1 \mathrm{~h}$ each at $37^{\circ} \mathrm{C}$. Tissue was then triturated and the dissociated cells were plated in DEM supplemented with $10 \% \mathrm{FBS}$ and $100 \mu \mathrm{g} / \mathrm{ml}$ penicillin and $100 \mu \mathrm{g} / \mathrm{ml}$ streptomycin.

ICR outbreed mice (Harlan Sprague-Dawley) were used for all experiments except those involving Bax-deficient, Apaf-1-deficient and p53-deficient sympathetic neurons. The genetic background of Bax-deficient, Apaf-1-deficient and p53deficient mice is C57BL/6; wild-type littermates were used as controls in these experiments. We note that the time course of TU-induced death of sympathetic neurons isolated from the $\mathrm{C} 57 \mathrm{BL} / 6$ genetic background is faster than in sympathetic neurons isolated from the ICR background. Bax-deficient mice were kindly provided by Dr. Stanley Korsmeyer and the Apaf-1-deficient mice were generated by Joachim Herz (UT Southwestern) and were kindly provided by the laboratory of Dr. Susan Ackerman (Jackson Laboratories). p53-deficient mice were purchased from Jackson Laboratories (Maine). Our methods for genotyping Bax-deficient mice has been described previously. ${ }^{28}$ The specific primers used to genotype Apaf-1- or p53-deficient mice are shown below:

\section{Apaf-1: Forward WT: 5' GCCTGCCATCCCATAGATGGT 3' Forward KO: 5' GATTGGGAAGACAATAGCAGG 3 Reverse (common): 5' CAGCAAGGCCTTTACCTGTTG $3^{\prime}$ WT: 900 bp; Apaf-1 KO: 600 bp \\ p53: $\quad$ Forward WT: $5^{\prime}$ ATAGGTCGGCGGTTCAT $3^{\prime}$ Reverse WT: $5^{\prime}$ CCCGAGTATCTGGAAGACAG $3^{\prime}$ Forward KO: $5^{\prime}$ TCCTCGTGCTTTACGGTATC $3^{\prime}$ Reverse KO: $5^{\prime}$ TATACTCAGAGCCGACCT $3^{\prime}$ WT: 600 bp; p53 KO: 280 bp}

Western blots. Western Blots were preformed as described previously. ${ }^{17}$ Primary antibodies were as follows: anti-CHOP/GADD-153 (sc-7351, Santa Cruz Biotechnology Inc.); anti-alpha tubulin (T9062, Sigma); anti-phospho-c-Jun (Ser 63) (9261, Cell Signaling Technology); anti-lactate dehydrogenase (200-1173, Rockland Immunochemicals Inc.). Mouse HRP-conjugated secondary antibody was purchased from Pierce Chemical $\mathrm{Co}$. Western blots were developed using the ECL-Plus detection system (Amersham Biosciences) and protein levels were quantified by scanning blots on a Typhoon scanner (Amersham Biosciences) and analyzed with ImageQuant software (Amersham Biosciences).

Quantitation of neuronal survival. Sympathetic neuronal survival after any treatment was assessed by counting clearly identifiable neurons with large and phase-bright cell bodies, whereas dead neurons atrophied and degenerated. All surviving cells in the culture were counted and expressed as a percent of the number of cells in the $\mathrm{Oh}$ condition. This method of assessing neuronal survival correlates well with other cell survival assays such as trypan blue exclusion and staining with calcein $\mathrm{AM}^{17}$

In order to determine the commitment of neurons to die, sympathetic neuronal survival, after varying lengths of TU treatment, was assessed by determining the percentage of cells that could be rescued by washing out the TU at those times. Sympathetic neurons plated in multiple dishes were treated with TU in the presence of NGF for the indicated times, rinsed three times and incubated in fresh NGF containing, TU-free media with or without CEP-11004 or SP600125 as indicated. Five to seven days after TU washout, the rescued neurons were clearly identifiable with large and phase-bright cell bodies, whereas the non-rescued neurons atrophied and degenerated. All rescued cells in the dish were counted and expressed as a percent of the number of cells in the $0 \mathrm{~h}$ condition.

Rate of MTT reduction. Culture media was removed and $500 \mu \mathrm{l} \mathrm{of} 0.4 \mathrm{mg} / \mathrm{ml}$. MTT in Leibovitz's L-15 medium containing $10 \%$ FBS was added to the neurons for $15 \mathrm{~min}$ at $37^{\circ} \mathrm{C}$. The MTT solution was aspirated and cells were lysed into $200 \mu \mathrm{l}$ dimethylsulfoxide. The amount of MTT formazan was quantitated by determining its absorbance at $490 \mathrm{nM}$ in a Bio-Tek Instuments Inc. ELx808 Ultra Microplate Reader. The same procedure was followed for MEFs except that MTT was in DMEM containing $10 \%$ FBS.

Immunofluroescence analysis. Immunofluorescence analysis on sympathetic neuronal cultures was performed as described previously. ${ }^{31}$ Cultures that were treated with NGF deprivation or TU addition were maintained in the presence of the pan caspase inhibitor $50 \mu \mathrm{M}$ zVAD-fmk to present cell loss. Primary antibodies were as follows: anti-cytochrome $c$ (556432, BD Biosciences) and antiphospho-c-Jun (Ser 63) (9261, Cell Signaling). Secondary antibodies used were anti-mouse Cy3-conjugated (Jackson Immunoresearch Laboratories, Inc., West Grove, PA) and anti-rabbit Alexa 488-conjugated (Molecular Probes Inc., Eugene, OR). Nuclei were stained with the nuclear dye bisbenzimide (Hoechst 33258 , Molecular Probes Inc., Eugene, OR)

Quantitative RT-PCR analysis. Our method of quantitative RT-PCR analysis is a modification of a previously published protocol, ${ }^{24}$ where we substituted the radioactivity-based detection method with a fluorescence-based detection technique. Briefly, RNA was isolated from sympathetic neuron cultures with Trizol (Invitrogen) using the manufactures protocol. Equal amounts of the RNA isolated at specific times after the specified treatment was converted into cDNA with 
SuperScript II Reverse Transcriptase (Invitrogen). Two microliters of cDNA was the template in a PCR using the following primer pairs:

BimEL: Forward 5' GGT AAT CCC GAC GGC GAA GGG AC 3'; Reverse 5' AAG AGA AAT ACC CAC TGG AGG ACC $3^{\prime}$

CHOP: Forward $5^{\prime}$ TCA CAC GCA CAT CCC AAA GC 3'; Reverse 5' TCA GCT

AGC TGT GCC ACT TTC C $3^{\prime}$

c-Jun: Forward 5' AAT GGG CAC ATC ACC ACT ACA C 3'; Reverse 5' TGC

TCG TCG GTC ACG TTC T $3^{\prime}$

Dp5: Forward 5' AGA CCC AGC CCG GAC CGA GCA A 3'; Reverse 5' CTC

TCT CTG TAG CTG GAC CTC 3

GAPDH: Forward 5' CCA TGG AGA AGG CTG GGG 3'; Reverse 5' CAA AGT

TGT CAT GGA TGA CC $3^{\prime}$

Noxa: Forward 5' GAA CGC GCC AGT GAA CCC AA 3'; Reverse 5' CTT TGT

CTC CAA TCC TCC GG $3^{\prime}$

Puma: Forward 5' CCT CAG CCC TCC CTG TCA CCA G $3^{\prime}$; Reverse $5^{\prime}$ CCG CCG CTC GTA CTG CGC GTT G 3

Primers and PCR protocol for XBP-1 were described previously. ${ }^{40}$ Primers for BiP were described previously. ${ }^{19}$ Preliminary experiments with sympathetic neuronal cultures validated that the RT-PCR technique was linear with respect to the amount of input RNA used for RT and with respect to the amount of CDNA used for PCR in these experiments. No product was amplified when water was used as input for a PCR reaction. Results were repeated in at least three independent RNA preparations. Levels were quantified using SYBR Green I Nucleic Acid Gel Stain (Molecular Probes Inc., Eugene, OR) and scanning blots on a Typhoon scanner (Amersham Biosciences) and analyzed with ImageQuant software (Amersham Biosciences).

Acknowledgements. We thank Kevin Wright, Allyson Vaughn and Yolanda Huang for critical review of this manuscript. We also thank Cephalon Inc. for kindly providing us the CEP-11004 compound for these studies. This work was supported by National Institutes of Health Grants NS42197 (to MD).

1. Wu J, Kaufman RJ. From acute ER stress to physiological roles of the unfolded protein response. Cell Death Differ 2006; 13: 374-384.

2. Lindholm D, Wootz H, Korhonen L. ER stress and neurodegenerative diseases. Cell Death Differ 2006; 13: 385-392.

3. Denault JB, Salvesen GS. Caspases: keys in the ignition of cell death. Chem Rev 2002 102: $4489-4500$.

4. Ham J, Towers E, Gilley J, Terzano S, Randall R. BH3-only proteins: key regulators of neuronal apoptosis. Cell Death Differ 2005; 12: 1015-1020.

5. Wang X. The expanding role of mitochondria in apoptosis. Genes Dev 2001; 15: 2922-2933.

6. Zong WX, Li C, Hatzivassiliou G, Lindsten T, Yu QC, Yuan J et al. Bax and Bak can localize to the endoplasmic reticulum to initiate apoptosis. J Cell Biol 2003; 162: 59-69.

7. Scorrano L, Oakes SA, Opferman JT, Cheng EH, Sorcinelli MD, Pozzan T et al. BAX and BAK regulation of endoplasmic reticulum Ca2+: a control point for apoptosis. Science 2003; 300: 135-139.

8. Nutt LK, Chandra J, Pataer A, Fang B, Roth JA, Swisher SG et al. Bax-mediated $\mathrm{Ca}^{2+}$ mobilization promotes cytochrome $c$ release during apoptosis. J Biol Chem 2002; 277: 20301-20308.

9. Nakagawa T, Zhu H, Morishima N, Li E, Xu J, Yankner BA et al. Caspase-12 mediates endoplasmic-reticulum-specific apoptosis and cytotoxicity by amyloid-beta. Nature 2000 403: 98-103.

10. Rao RV, Castro-Obregon S, Frankowski H, Schuler M, Stoka V, del Rio G et al. Coupling endoplasmic reticulum stress to the cell death program. An Apaf-1-independent intrinsic pathway. J Biol Chem 2002; 277: 21836-21842.

11. Morishima N, Nakanishi K, Takenouchi H, Shibata T, Yasuhiko Y. An endoplasmic reticulum stress-specific caspase cascade in apoptosis. Cytochrome $c$-independen activation of caspase-9 by caspase-12. J Biol Chem 2002; 277: 34287-34294

12. Fischer $\mathrm{H}, \mathrm{Koenig} U$, Eckhart L, Tschachler $E$. Human caspase 12 has acquired deleterious mutations. Biochem Biophys Res Commun 2002; 293: 722-726.

13. Saleh M, Mathison JC, Wolinski MK, Bensinger SJ, Fitzgerald P, Droin N et al. Enhanced bacterial clearance and sepsis resistance in caspase-12-deficient mice. Nature 2006; 440 1064-1068

14. Di Sano F, Ferraro E, Tufi R, Achsel T, Piacentini M, Cecconi F. Endoplasmic reticulum stress induces apoptosis by an apoptosome-dependent but caspase 12-independent mechanism. J Biol Chem 2006; 281: 2693-2700.
15. Reimertz C, Kogel D, Rami A, Chittenden T, Prehn JH. Gene expression during ER stress-induced apoptosis in neurons: induction of the $\mathrm{BH} 3$-only protein Bbc3/PUMA and activation of the mitochondrial apoptosis pathway. J Cell Biol 2003; 162: 587-597.

16. Chang JY, Korolev VV. Specific toxicity of tunicamycin in induction of programmed cell death of sympathetic neurons. Exp Neurol 1996; 137: 201-211.

17. Potts PR, Singh S, Knezek M, Thompson CB, Deshmukh M. Critical function of endogenous XIAP in regulating caspase activation during sympathetic neuronal apoptosis. $J$ Cell Biol 2003; 163: 789-799.

18. Urano F, Wang X, Bertolotti A, Zhang Y, Chung P, Harding HP et al. Coupling of stress in the ER to activation of JNK protein kinases by transmembrane protein kinase IRE1. Science 2000; 287: 664-666.

19. Nishitoh H, Matsuzawa A, Tobiume K, Saegusa K, Takeda K, Inoue K et al. ASK1 is essential for endoplasmic reticulum stress-induced neuronal cell death triggered by expanded polyglutamine repeats. Genes Dev 2002; 16: 1345-1355.

20. Maroney AC, Finn JP, Bozyczko CD, O'Kane TM, Neff NT, Tolkovsky AM et al. CEP-1347 (KT7515), an inhibitor of JNK activation, rescues sympathetic neurons and neuronally differentiated PC12 cells from death evoked by three distinct insults. J Neurochem 1999; 73: 1901-1912

21. Wang LH, Paden AJ, Johnson Jr EM. Mixed-lineage kinase inhibitors require the activation of Trk receptors to maintain long-term neuronal trophism and survival. J Pharmacol Exp Ther 2005; 312: 1007-1019.

22. Mota M, Reeder M, Chernoff J, Bazenet CE. Evidence for a role of mixed lineage kinases in neuronal apoptosis. J Neurosci 2001; 21: 4949-4957.

23. Xu Z, Maroney AC, Dobrzanski P, Kukekov NV, Greene LA. The MLK family mediates cJun N-terminal kinase activation in neuronal apoptosis. Mol Cell Biol 2001; 21: 4713-4724.

24. Estus S, Zaks WJ, Freeman RS, Gruda M, Bravo R, Johnson Jr EM. Altered gene expression in neurons during programmed cell death: identification of c-jun as necessary for neuronal apoptosis. J Cell Biol 1994; 127: 1717-1727.

25. Palmada M, Kanwal S, Rutkoski NJ, Gustafson-Brown C, Johnson RS, Wisdom R et al. cjun is essential for sympathetic neuronal death induced by NGF withdrawal but not by $p 75$ activation. J Cell Biol 2002; 158: 453-461.

26. Murakata C, Kaneko M, Gessner G, Angeles TS, Ator MA, O'Kane TM et al. Mixed lineage kinase activity of indolocarbazole analogues. Bioorg Med Chem Lett 2002; 12: 147-150.

27. Sun YF, Yu LY, Saarma M, Timmusk T, Arumae U. Neuron-specific Bcl-2 homology 3 domain-only splice variant of Bak is anti-apoptotic in neurons, but pro-apoptotic in nonneuronal cells. J Biol Chem 2001; 276: 16240-16247.

28. Deckwerth TL, Elliott JL, Knudson CM, Johnson Jr EM, Snider WD, Korsmeyer SJ. Bax is required for neuronal death after trophic factor deprivation and during development. Neuron 1996: 17: 401-411.

29. Besirli CG, Deckwerth TL, Crowder RJ, Freeman RS, Johnson Jr EM. Cytosine arabinoside rapidly activates Bax-dependent apoptosis and a delayed Bax-independent death pathway in sympathetic neurons. Cell Death Differ 2003; 10: 1045-1058.

30. Hetz C, Bernasconi P, Fisher J, Lee AH, Bassik MC, Antonsson B et al. Proapoptotic BAX and BAK modulate the unfolded protein response by a direct interaction with IRE1alpha. Science 2006; 312: 572-576.

31. Deshmukh M, Kuida K, Johnson Jr EM. Caspase inhibition extends the commitment to neuronal death beyond cytochrome $c$ release to the point of mitochondrial depolarization. $J$ Cell Biol 2000; 150: 131-143.

32. Breckenridge DG, Germain M, Mathai JP, Nguyen M, Shore GC. Regulation of apoptosis by endoplasmic reticulum pathways. Oncogene 2003; 22: 8608-8618.

33. Harding TC, Xue L, Bienemann A, Haywood D, Dickens M, Tolkovsky AM et al. Inhibition of JNK by overexpression of the JNK binding domain of JIP-1 prevents apoptosis in sympathetic neurons. J Biol Chem 2001; 276: 4531-4534.

34. Imaizumi K, Benito A, Kiryu-Seo S, Gonzalez V, Inohara N, Lieberman AP et al. Critical role for DP5/Harakiri, a Bcl-2 homology domain 3-only Bcl-2 family member, in axotomyinduced neuronal cell death. J Neurosci 2004; 24: 3721-3725.

35. Wyttenbach A, Tolkovsky AM. The BH3-only protein Puma is both necessary and sufficient for neuronal apoptosis induced by DNA damage in sympathetic neurons. J Neurochem 2006; 96: 1213-1226

36. Luo $X, H e Q$, Huang $Y$, Sheikh MS. Transcriptional upregulation of PUMA modulates endoplasmic reticulum calcium pool depletion-induced apoptosis via Bax activation. Cell Death Differ 2005; 12: 1310-1318.

37. Levine B, Yuan J. Autophagy in cell death: an innocent convict? J Clin Invest 2005; 115: 2679-2688.

38. Shiraishi H, Okamoto H, Yoshimura A, Yoshida H. ER stress-induced apoptosis and caspase-12 activation occurs downstream of mitochondrial apoptosis involving Apaf-1. J Cell Sci 2006; 119: 3958-3966.

39. Obeng EA, Boise LH. Caspase-12 and caspase-4 are not required for caspase-dependent endoplasmic reticulum stress-induced apoptosis. J Biol Chem 2005; 280: 29578-29587.

40. Harding HP, Zhang Y, Khersonsky S, Marciniak S, Scheuner D, Kaufman RJ et al. Bioactive small molecules reveal antagonism between the integrated stress response and sterol-regulated gene expression. Cell Metab 2005; 2: 361-371. 